\title{
Perturbation Maps and the ring of Haumea
}

\author{
Diogo M. Sanchez ${ }^{\oplus},{ }^{1 \star}$ Rogerio Deienno, ${ }^{2 \star}$ Antonio F. B. A. Prado ${ }^{1 \star}$ \\ and Kathleen C. Howell ${ }^{3 \star}$ \\ ${ }^{1}$ National Institute for Space Research, Av. dos Astronautas, 1758, São José dos Campos, SP 12227-010, Brazil \\ ${ }^{2}$ Department of Space Studies, Southwest Research Institute, 1050 Walnut Street, Boulder CO 80302, USA \\ ${ }^{3}$ School of Aeronautics and Astronautics, Purdue University, 701 West Stadium Avenue, West Lafayette, IN 47907-2045, USA
}

Accepted 2020 June 8. Received 2020 May 28; in original form 2019 March 1

\begin{abstract}
The dwarf planet Haumea is a trans-Neptunian object that is orbited by two moons and has a recently discovered ring. The particles of this ring are near the 3:1 resonance between the spin of Haumea and the orbital motion of the particles. In this work, the ring of Haumea is investigated using Perturbation Maps. These maps show the behaviour and impact of perturbations acting over particles around Haumea. The information coming from the maps depends on the integral type for the disturbing acceleration used to build the maps. The types II and IV are used. The numerical simulations are focused in the region between 2000 and $2500 \mathrm{~km}$ from the centre of Haumea, which is the region where the ring was observed, considering two initial values for the 3:1 resonant angle: $\theta_{\text {res }}=0^{\circ}$ and $\theta_{\text {res }}=270^{\circ}$. The possible stable region for the initial angle $\theta_{\text {res }}=0^{\circ}$ is larger than the stable region for the initial angle $\theta_{\text {res }}=270^{\circ}$. Furthermore, we found that these stable regions are not continuous, indicating that there are possible gaps in the ring. Therefore, our results suggest that Haumea may not have only one single ring, but a system of rings instead. Possible transit of the particles between the ring and the region close to the orbit of Namaka is also shown.
\end{abstract}

Key words: celestial mechanics - minor planets, asteroids: individual: Haumea-planets and satellites: rings.

\section{INTRODUCTION}

Haumea is a trans-Neptunian Object (TNO) with the shape of a triaxial ellipsoid in fast rotation $(3.9155 \mathrm{~h})$. It possesses a rocky core covered by a thin layer of carbon depleted ice (Pinilla-Alonso et al. 2009). Haumea is also orbited by two moons, Namaka and Hi' iaka (Ragozzine \& Brown 2009). The inner moon Namaka has considerable eccentricity $(0.249)$ and it is inclined with respect to the equator of Haumea $\left(13^{\circ}\right)$. Alternatively, the outer moon Hi'iaka has a near circular orbit (eccentricity equal to 0.051 ) and low inclination $\left(1^{\circ}-2^{\circ}\right)$, also with respect to the equator of Haumea (Ćuk, Ragozzine \& Nesvorný 2013). These characteristics, added to the possibility that Haumea is the parent body of a collisional family (Levison et al. 2008; Desch \& Neveu 2015), make this dwarf planet one of the most intriguing objects in the Solar system. Recently one more characteristic was added to this system: the discovery of a ring around Haumea (Ortiz et al. 2017). This ring was discovered in a region near the 3:1 resonance between the spin of Haumea and the mean motion of the particles of the ring.

The Haumea system possesses another particular characteristics. There is a chaotic region between Haumea and Namaka (Sanchez \&

^E-mail: diogo.sanchez@inpe.br (DMS); rdeienno@boulder.swri.edu (RD); antonio.prado@inpe.br (AP); howell@purdue.edu (KH)
Prado 2017), caused by the superposition of the critical semimajor axis of the pairs Haumea/Namaka and Haumea/Hi'iaka. This relationship implies that any particle that leaves the ring region by increasing its the semimajor axis and/or eccentricity may cross the chaotic region and could be ejected from the system. There is also the possibility of collisions with Hi'iaka. These findings indicate that, even if the perturbation of Namaka and Hi' iaka is negligible for orbits close to Haumea, as claimed by Sicardy et al. (2018), and confirmed by our preliminary simulations, they need to be incorporated in the simulations of the ring.

In the past few years, the dynamics of particles and spacecraft in the Haumea system have been analysed by the authors in several different ways. For instance: by separating the system in two pairs, Haumea/Namaka and Haumea/Hi'iaka, by using the formalism of the Circular Restricted Three-Body Problem (Sanchez, Prado \& Yokoyama 2016a), considering the Restricted Full Three Body Problem (Sanchez, Howell \& Prado 2016b, 2017), and using the direct integration of the Newtonian equations of motion of the system, considering several disturbers (Sanchez \& Prado 2017). Particularly, in Sanchez et al. (2016b), a region with direct stable orbits was found. Since, at the time of that publication, no ring was observed, the authors came to the conclusion that the apparently stable region found was useful to place a spacecraft to explore the system. However, in 2017, after a large number of observations, a $70 \mathrm{~km}$ wide ring was discovered around Haumea. This ring has orbital radius of 
Table 1. Some parameters of the Haumea system.

\begin{tabular}{lccccc}
\hline Object & Mass, kg & $a, \mathrm{~km}$ & $e$ & $I, \mathrm{deg}$ & Orbital period \\
\hline Haumea & $4.006 \times 10^{21}$ & $6.46020 \times 10^{9}$ & 0.19368 & 28.22281 & $283.28 \mathrm{yr}$ \\
Namaka & $1.79 \times 10^{18}$ & 25657 & 0.249 & 113.013 & $18.27 \mathrm{~d}$ \\
Hi'iaka & $1.79 \times 10^{19}$ & 49880 & 0.0513 & 126.356 & $49.44 \mathrm{~d}$ \\
\hline
\end{tabular}

approximately $2287 \mathrm{~km}$. Additionally, new values for the shape of Haumea (Ortiz et al. 2017) were determined. Comparing the results from Sanchez et al. (2016b) with the actual position of the ring, one can conclude that the possible stable region found in that paper is actually the region of the ring, considering the previous shape of Haumea suggested by Lockwood, Brown \& Stansberry (2014).

In this context, this paper aims to investigate the dynamics of particles around Haumea, in the region where the ring of Haumea was found, i.e., particles with semimajor axis from $2000 \mathrm{~km}$ to $2500 \mathrm{~km}$. To investigate the behaviour of these particles, we use Perturbation Maps (Sanchez \& Prado 2019). These maps are grids of initial conditions that show how much the perturbations, coming from several sources in the system, such as those from the moons of Haumea, affect the orbital motion of these particles. The information in the maps is produced via the method of the integral of the disturbing accelerations (Prado 2013, 2014).

The method of the integral of the disturbing accelerations, or PI method, was conceived to allow a quantitative assessment of the mean perturbation that acts over a spacecraft path. The concept behind the method is that the integral of the acceleration from a disturber gives the mean variation of the velocity of the spacecraft (a massless particle in the context of this paper) due to this disturber, for a given period of time $T$. In other words, the method measures the perturbation from a disturber, or a set of disturbers, in terms of a mean variation of the velocity of the particle. Since its first appearance (Prado 2013), the method has evolved and improved (Carvalho, de Moraes \& Prado 2014; Oliveira \& Prado 2014; Sanchez, Prado \& Yokoyama 2014; dos Santos et al. 2015; Venditti \& Prado 2015; Lara 2016; Sanchez \& Prado 2017; de Almeida et al. 2018). There are four types of integrals: the integral of the absolute value of the disturbing accelerations (type I), which is the original method; the integral of the disturbing accelerations in the direction of the velocity of the disturbed body (type II); the integral of the disturbing accelerations without the modulus (type III); and the integral of the difference between the acceleration of the disturbed body and a reference orbit (type IV). A grid of initial conditions evaluated via the integral of the disturbing acceleration is called a Perturbation Map. For the particles that compose the ring of Haumea, an analysis based on Perturbation Maps can reveal the possible bias of the stability of these particles. This means that the maps can reveal unstable behaviour of the particles in the ring, as well as possible stable behaviours, even for low values of $T$. The indication of long-term stability given by the maps can be confirmed by long-term integrations.

This work is organized as follows. In Section 2, we explain the numerical model used in the paper and the PI method, with its four variations and the physical meaning of each one. In Section 3, we present the results of all numerical simulations, Perturbation Maps, long term simulations, and some considerations about the ring structure and the resonance in the ring. Section 4 concludes the paper.

\section{MATHEMATICAL MODELS AND METHODS}

The numerical model used to simulate the motion of the particles in the ring of Haumea is presented in this section. The PI method and the physical meaning of the Perturbation Maps are also explained. Table 1 presents some physical and orbital parameters of the Haumea system (Ragozzine \& Brown 2009), such as semimajor axis $(a)$, eccentricity $(e)$, and inclination $(I)$, to be used in the next sections. The value of the semimajor axis of Haumea corresponds to approximately $43 \mathrm{au}$, where $1 \mathrm{au}=1.49597870700 \times 10^{8} \mathrm{~km}$ is the astronomical unit used in this paper. The inclinations in this table are given with respect to the Ecliptic frame. In the case of Namaka and Hi'iaka, the corresponding inclinations with respect to the equator of Haumea are, respectively, $13^{\circ}$ and $2^{\circ}$ (Ćuk et al. 2013).

\subsection{Numerical model}

The equation of motion (EOM) of a particle that comprises the ring of Haumea is given by

$\ddot{\boldsymbol{r}}=-\frac{G M_{\mathrm{H}}}{|\boldsymbol{r}|^{3}} \boldsymbol{r}+G \sum_{j=1}^{N-1} M_{j}\left(\frac{\boldsymbol{r}_{j}-\boldsymbol{r}}{\left|\boldsymbol{r}_{j}-\boldsymbol{r}\right|^{3}}-\frac{\boldsymbol{r}_{j}}{\left|\boldsymbol{r}_{j}\right|^{3}}\right)+\boldsymbol{P}_{\mathrm{H}}$,

where $_{\mathrm{I}}$ is the universal gravitational constant, $M_{\mathrm{H}}, M_{j}$, and $\boldsymbol{r}_{j}$ are the mass of Haumea and the masses and position vectors of the disturbers (Hi'iaka, Namaka, and the Sun), respectively. $\boldsymbol{r}$ is the position vector of the particle. The reference system is centred in Haumea, with a fundamental reference plane in the equator of Haumea, aligned with the I-axis at the reference epoch. The equator of Haumea is defined as the plane perpendicular to its spin axis. $\boldsymbol{P}_{\mathrm{H}}$ is the acceleration due to the gravitational potential of Haumea, expanded in spherical harmonics up to degree and order four. This potential is calculated using a modified recursive model given by Sanchez et al. (2014), adapted to Haumea. The adaptations were necessary because this model was originally defined for the Earth, and it depends on the Greenwich Sidereal Time (GST), which only makes sense for the Earth. To adapt this model to Haumea, an angle that starts at the $x$-axis was defined, which is fixed as the larger axis for the shape of Haumea. The initial value of this angle is always zero. Due to the symmetry of Haumea (a triaxial ellipsoid) all odd coefficients of the spherical harmonics expansion are zero. Thus, the remaining coefficients of the spherical harmonics expansion up to degree and order four were calculated (Balmino 1994) considering the shape of Haumea found by Ortiz et al. (2017), which is $a=1$, $161 \mathrm{~km}, b=852 \mathrm{~km}$, and $c=513 \mathrm{~km}$, where $a, b, c$ are the semiaxes of Haumea assumed as a triaxial ellipsoid. The coefficients are

$$
\begin{aligned}
& C_{20}=-0.1148054670859791, \\
& C_{22}=0.2307319939373301 \times 10^{-1}, \\
& C_{40}=0.3052508642861946 \times 10^{-1}, \\
& C_{42}=-0.1892092452546749 \times 10^{-2}, \\
& C_{44}=0.9506652326124315 \times 10^{-4} .
\end{aligned}
$$

For completeness, the EOM for Hi'iaka and Namaka is 


$$
\begin{aligned}
\ddot{\boldsymbol{r}}_{\mathrm{NS}}= & -\frac{G\left(M_{\mathrm{H}}+M_{\mathrm{NS}}\right)}{\left|\boldsymbol{r}_{\mathrm{NS}}\right|^{3}} \boldsymbol{r}_{\mathrm{NS}} \\
& +G \sum_{j=1}^{N-1} M_{j}\left(\frac{\boldsymbol{r}_{j}-\boldsymbol{r}_{\mathrm{NS}}}{\left|\boldsymbol{r}_{j}-\boldsymbol{r}_{\mathrm{NS}}\right|^{3}}-\frac{\boldsymbol{r}_{j}}{\left|\boldsymbol{r}_{j}\right|^{3}}\right)+\boldsymbol{P}_{\mathrm{H}},
\end{aligned}
$$

where $M_{\mathrm{NS}}$ and $\boldsymbol{r}_{\mathrm{NS}}$ are the masses and position vectors of the natural satellites. We assume that Namaka and Hi' iaka are mutually disturbed and also disturbed by the gravitational potentials of Haumea and the Sun. Since we consider the Sun in a Keplerian orbit, its EOM is

$\ddot{\boldsymbol{r}}_{\odot}=-\frac{G\left(M_{\mathrm{H}}+M_{\odot}\right)}{\left|\boldsymbol{r}_{\odot}\right|^{3}} \boldsymbol{r}_{\odot}$,

where $M_{\odot}$ and $\boldsymbol{r}_{\odot}$ are the mass and the position vector of the Sun. We use the Epoch 2008, May 28th for the initial conditions for the massive bodies. For all numerical simulations, we use the RADAU (RA15) integrator (Everhart 1985).

\subsection{PI method and Perturbation Maps}

Perturbation Maps (or PMaps) are grids of initial conditions evaluated via the method of the integral of the disturbing accelerations, or simply the PI method. Each point in the map shows the value of the PI for a given initial condition. The PMap carries the name of the type of integral used to build it. For example, a PMap of type II is the one generated by using the integral of type II. There are four types of integrals, and each of them can reveal different aspects of the perturbations that act over a particle in the ring of Haumea. The four types of integral are

$$
\begin{aligned}
& \mathrm{PI}_{i}=\frac{1}{T} \int_{0}^{T}|\boldsymbol{a}| \mathrm{d} t, \\
& \mathrm{PI}_{\mathrm{ii}}=\frac{1}{T} \int_{0}^{T}\langle\boldsymbol{a}, \hat{\boldsymbol{v}}\rangle \mathrm{d} t, \text { with } \hat{\boldsymbol{v}}=\boldsymbol{v} /|\boldsymbol{v}|, \\
& \mathrm{PI}_{\mathrm{iii}}=\frac{1}{T}\left|\int_{0}^{T} \boldsymbol{a} \mathrm{d} t\right|, \\
& \mathrm{PI}_{\mathrm{iv}}=\frac{1}{T}\left|\int_{0}^{T} \ddot{\boldsymbol{r}}-\ddot{\boldsymbol{r}}_{k} \mathrm{~d} t\right|,
\end{aligned}
$$

where $\boldsymbol{a}$ can be interpreted as either the acceleration due to each disturber, or the summation of all disturbing accelerations. The latter applies to our case, where $\boldsymbol{a}$ denotes the summation of the second and third terms of equation (1). $v$ is the velocity of the particle. $\ddot{\boldsymbol{r}}_{k}$ is the acceleration of a particle in a Keplerian orbit with the same initial conditions of the particle analysed. $T$ is the final time of the numerical integration for the trajectory of the particle, which can be either the total time of the numerical integration or the time until a critical event, as a collision, that stops the integration before the total time. Since $T$ can acquire any value between the initial time and the total time of integration, the integrals of the PI method are normalized by $\frac{1}{T}$. Thus, the unit of the final value of the PI will always be velocity per unit of time. The normalization is necessary to avoid misinterpretation when comparing orbits with different durations.

The first type of integral measures the total perturbation applied to the particle. When the acceleration due to a perturbation is symmetric with respect to one orbital period, the effects caused by this perturbation can be compensated. An example is the solar radiation pressure. Let us consider a satellite around the Earth, in a circular orbit parallel to the Ecliptic. In one orbital period, the amount of energy that the satellite loses, due to the solar radiation pressure, when moving towards the Sun, is regained when this satellite is moving away from the Sun. Thus, over one orbital period, this specific orbit returns to the same point of the origin. However, this fact does not imply that the orbit is not disturbed, since the trajectories with solar radiation pressure and without this effect are not the same. Then, the first type of integral, using the absolute value of the acceleration, allows us to compare forces, even forces with a different nature, because this type of integral eliminates compensation of the effects of the perturbations. Therefore, it is useful only when it is desired to consider effects during a single orbital period, and not for the long term evolution of the orbit. For this reason this type of integral is not applied in this work.

The integral of the second type shows the perturbations that directly affect the variation of energy along the orbit of the particle. If the value of this integral is positive, it means that the perturbation 'added' energy to the orbit of the particle, raising its semimajor axis. In the opposite situation, when the value of this type of integral is negative, it decreases its semimajor axis. This type of integral is also useful to assess the stability of a particle orbit. For instance, if the orbits of two particles, with a small difference in initial conditions, have different signs in the value of the $\mathrm{PI}_{\mathrm{ii}}$, it means that there is a strong possibility that these particles are inside a chaotic region around the central body. This interpretation relies on the fact that these two orbits, although close to each other, have very different behaviour. This property provides an assessment of the chaotic behaviour in the system. This type of integral is one of the two types that will be used to study the dynamics of the particles of the ring of Haumea. This type of integral allows compensation of the effects along the trajectory.

The third type of integral measures the mean perturbation applied to the particle. Since this type of integral is calculated by using $\boldsymbol{a}$, it allows compensational effects and it is useful to compare forces from disturbers of similar nature, for example, the third body perturbation of the Sun and a moon of Haumea. It is also useful to identify and separate perturbations coming from different sources. As the integral of the first type, this one is calculated over one orbital period. For these reasons, this integral is not used in this work.

The fourth type of PI is actually a variation of the third type. It was idealized in Lara (2016), and later used in de Almeida et al. (2018). Since this type is the integral of the difference between the total acceleration of the disturbed particle and the acceleration of a particle in a reference orbit (a Keplerian orbit in this paper) with the same initial conditions as the disturbed one, it measures how much the disturbed orbit deviates from the reference orbit. Considering that the dynamics in the Cartesian system reflect the dynamics in orbital elements and vice versa, any variation in the orbital elements will result in an increase in the value of $\mathrm{PI}_{\mathrm{iv}}$. Therefore, this is the other type of $\mathrm{PI}$, along with $\mathrm{PI}_{\mathrm{ii}}$, that is used to build the perturbation maps to study the ring of Haumea in this work.

\section{RESULTS}

Equation (1), as well as the equations of motion of the remaining bodies, are numerically integrated using the RADAU integrator, hereafter called the main simulation. Preliminary tests demonstrated that each trajectory needs between 2500 and 10000 points to ensure the accuracy of the PI method and, consequently, the accuracy of the PMaps. The number of points increases when the orbit is close to the central body. Thus, since the region analysed in this work is the region between 2000 and $2500 \mathrm{~km}$ from Haumea, the number of points in each orbit was kept at 10000 . To keep this number of points, even for integrations that stop before the final time due to critical events like collision, resulting in a number of points less than the desired one, the program re-runs the simulation adjusting the time-steps to ensure 10000 points before the critical event. 
To build the PMaps, all the accelerations are stored in temporary files during the main simulation. After the end of the main simulation, the code uses the temporary files to calculate the PI, using the Simpson 1/3 method (Burden \& Faires 2010). This strategy is necessary to avoid the inclusion of accelerations coming from the sub-steps of the RADAU integrator, which uses a variable stepsize, and to retain the constant spacing for the PI method. This also ensures the accuracy of the method and saves computational time. Another important point, as the concept behind the PMaps suggests, is that the result in the map is the average velocity, per unit of time, that the particle receives from the disturber. Since this value is averaged, the initial Epoch does not affect the conclusions of the work.

The time of integration, $T$, is another important factor for the effectiveness in the implementation of the PMaps. The longer is the time of integration, the longer is the interaction between the particle and the disturbers. Therefore, the longer is the time of integration, the higher will be the value of the integral in the PI method. However, since the integral of the PI method is normalized by $1 / T$, the value of the PI is not influenced by the time of the integration, but the structures that the PMaps can reveal are influenced by $T$. To explain this, let us suppose that a particle is orbiting Haumea, at a semimajor axis of $20000 \mathrm{~km}$, which is close to Namaka. For a PMap with $T=$ $t_{1}$, this particle may not escape or collide, staying around Haumea. In this PMap, a point may not reveal a high value of PI, because this time interval is not sufficient large to show the whole impact of Namaka over the particle, if $t_{1}$ is less than the orbital period of Namaka. However, if we take $T=t_{2}$, with $t_{2}>t_{1}$, the time may allow Namaka to interact with the particle and, consequently, the particle would have a high value of PI, changing the 'face' of the PMap. For any system, there is a time $T=t_{n}$ for which the PMap resists further change. This time is called the saturation time and it is related to the repetition of the system geometry. Then, the saturation time depends on the nature and number of disturbers. Since the dependency on $T$ in the PMap is related to third body perturbations as well as the repetition of the geometry of the external disturbers, the saturation time can be estimated by comparing the time of integration with the period of the slowest angular variable in the system to achieve the goal of the map.

In the case of the analysis of the ring of Haumea, preliminary simulations showed that the influence of the moons of Haumea is not relevant, because the ring is too close to Haumea, whose perturbation from its gravitational potential overcomes the remaining perturbations from the system. However, when a particle leaves the ring, an interaction with the moons is possible, but this always occurs for short times. For this reason, the time of saturation is considerably short; with $T=1 \mathrm{yr}$ the maps saturate. Thus, with this value of $T$, the PMaps can show the tendency of stability or instability in the orbits of the particles and remain the same, regardless how much $T$ is increased. Although preliminary tests showed that the influence of the Sun in not considerable, to keep the consistency of the simulations and to allow the comparison of the PMaps with the long-term integrations, the Sun is incorporated in the simulations, as explained in the previous section.

\subsection{Regular grid of initial conditions}

To start the analysis of the system, a regular grid of initial semimajor axis, $a_{0}$, and initial eccentricity, $e_{0}$, was built. The grid illustrates the maximum eccentricity achieved in $1 \mathrm{yr}$ of integration. It includes almost planar orbits $\left(I=0.001^{\circ}\right)$, with initial longitude of ascending node $(\Omega)$ and mean anomaly $(l)$ equal to zero. The initial value of the argument of the perigee $(\omega)$ depends on the desirable initial value of the resonant angle.

According to Sicardy et al. (2018), the order of the resonance and, consequently, the initial value of the resonant angle, will depend on the gravitational model. If an ellipsoid shape is considered for the gravitational model, the resonance only appears in its fourthorder version (6:2 resonance instead of 3:1 resonance). Such is the case of our model, where a triaxial ellipsoid shape is considered in the calculation of the spherical harmonics. However, for the same gravitational potential, these resonances are essentially the same. Thus, to define the initial value of $(\omega)$, we chose the $3: 1$ resonant angle $\theta_{\text {res }}=\lambda_{\mathrm{H}}-3 \lambda+2 \omega$, where $\lambda_{\mathrm{H}}$ is the rotation angle of Haumea, $\lambda$ and $\varpi$ are, respectively, the mean longitude and the longitude of the pericentre of the particles. Another implication of the ellipsoidal shape is that the gravitational potential is invariant under a rotation about $\pi$. Consequently, there are two configurations that represent the 3:1 spin-orbit resonance: $\theta_{\text {res }}= \pm 90^{\circ}$. These two configurations are symmetrical, thus, we chose only the $\theta_{\text {res }}=270^{\circ}$ to represent initial conditions at the centre of the 3:1 resonance. We also considered $\theta_{\text {res }}=0^{\circ}$ as a configuration outside the libration centre of the resonance to compare resonant and non-resonant configurations. Therefore, for $\theta_{\text {res }}=0^{\circ}$, we start $\omega$ at $0^{\circ}$, and to produce a result for $\theta_{\text {res }}=270^{\circ}$, we start $\omega$ at $90^{\circ}$.

There are three final scenarios for the orbit of the particle: (i) the particle collides with Haumea, Namaka, or Hi'aka; (ii) the particle escapes from the system; and (iii) the particle remains around Haumea during the time of integration. In the first scenario, we consider collision with Haumea when the particle is inside a sphere of radius equal to the largest axis of Haumea, $1161 \mathrm{~km}$. In the case of collision with Namaka or Hi'iaka, the logic is the same, but with radii equal to 100 and $195 \mathrm{~km}$, respectively. However, due to the small size of the moons, collision with either Namaka or Hi'iaka is rare, although there are close encounters between the particles and Namaka. In the second scenario, an escape occurs when the distance of the particle, with respect to Haumea, achieves $4.6 \times 10^{6} \mathrm{~km}$, which is the approximate value of the Hill radius of Haumea.

Regular grids are shown in Fig. 1(a), for $\theta_{\text {res }}=0^{\circ}$, and Fig. 1(b), for $\theta_{\text {res }}=270^{\circ}$. In Fig. 1(a), there are three noticeable regions: the first one between $a_{0}=2000$ and $a_{0} \approx 2100 \mathrm{~km}$, the second one from $a_{0} \approx 2100$ to $a_{0} \approx 2350 \mathrm{~km}$, and the third one from $a_{0} \approx 2350$ to $a_{0} \approx 2500 \mathrm{~km}$. These regions are comprised of orbits that keep low values of maximum eccentricity during the maximum time of integration $(1 \mathrm{yr})$. The remaining part of the figure include orbits that escape from the system, whose maximum eccentricity achieves values near one, and orbits that collided with Haumea, Namaka, or Hi' iaka (white region) after some revolutions. The white region also represents orbits that have an initial periapsis radius inside Haumea. There is no distinction between the bodies with which the particle collides. In Fig. 1(b), there are regions that stand out, but in the case of this figure, these regions are more distinct. Comparing Fig. 1(a) with Fig. 1(b), one can see that the regions with orbits that retain low values of maximum eccentricity are concentrated in areas with low values of initial eccentricity in Fig. 1(a), whereas the correspondent region in Fig. 1(b) is concentrated in higher values of initial eccentricity.

Similar to Fig. 1, Fig. 2 shows the survival time of each particle as a function of $a_{0}$ and $e_{0}$. Fig. 2 confirms the findings in Fig. 1, where particles with larger values of maximum eccentricity are short lived. However, the information coming from these two figures is limited. There is no information about the mechanism behind the 


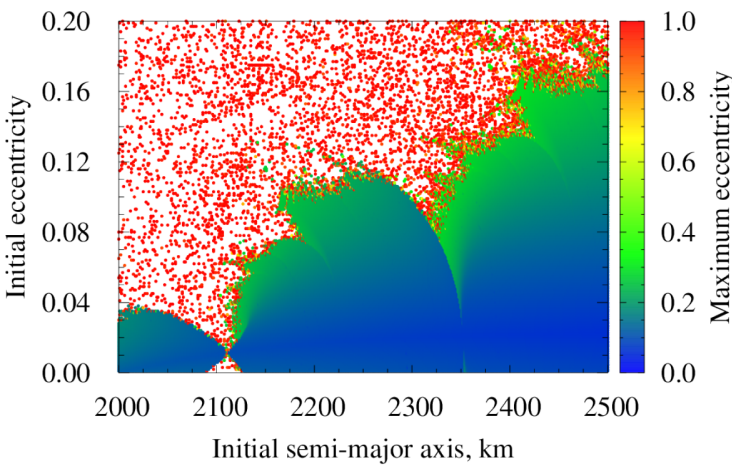

(a) $\theta_{\text {res }}=0^{\circ}$

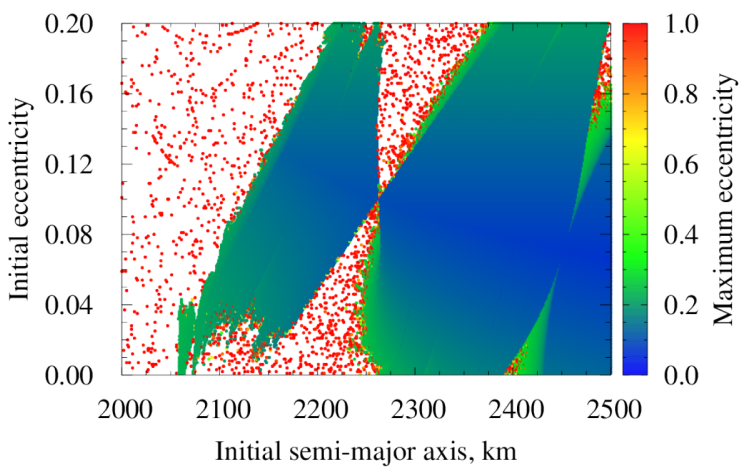

(b) $\theta_{\text {res }}=270^{\circ}$

Figure 1. Maximum eccentricity achieved by particles around Haumea as a function of the initial semimajor axis and eccentricity. Initial inclination $I=0.001^{\circ} . \varpi_{0}=0^{\circ}$ (a) and $\varpi_{0}=90^{\circ}$ (b). Maximum time of integration equal to $1 \mathrm{yr}$. The white region stands for orbits that collided with a body in the system after some revolutions and orbits that have initial periapsis radius inside Haumea.

growth in eccentricity, and there is no way to make long term predictions by analysing the behaviour of the particles in $1 \mathrm{yr}$ with these grids. For this reason, it is necessary that the analysis of the PMaps is evaluated along with the integrations that generated Figs 1 and 2 .

\subsection{PMaps}

In a PMap there are high values of PI where critical events occur. These critical events can be collisions or escapes. The difference between the high and low values of PI in the same map can generate problems in the visualization of fine structures in the map. To avoid problems in visualisation, we constrained the scale of the maps to allow the visualisation of these fine structures. Consequently, regions in the maps that present values in the extremes of the colour bar scale may have higher values of PI. The same logic is applied to negative minimum values of PI, especially in the case of the PMap of type II.

This section is separated into three parts. In the first part, the PMap built with the PI method of type II is presented considering all perturbations combined. Next we present the individual contribution of each coefficient of the spherical harmonics in the gravitational potential of Haumea. Lastly, the PMap of type IV is presented. It is important to highlight that all these maps were calculated along with the main integration, presented in the previous section,

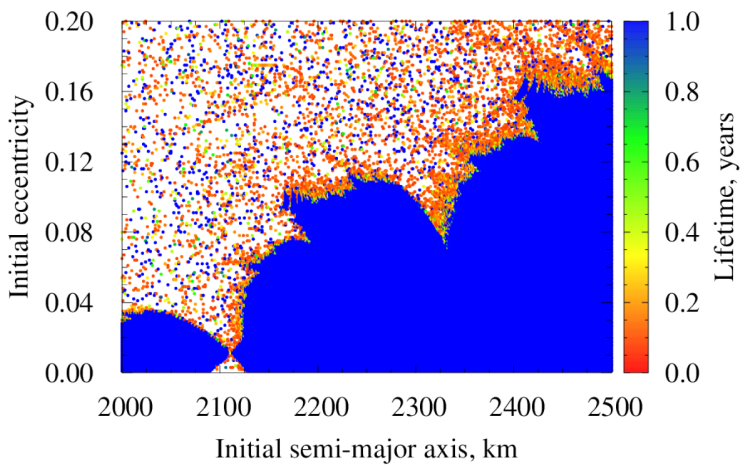

(a) $\theta_{\text {res }}=0^{\circ}$

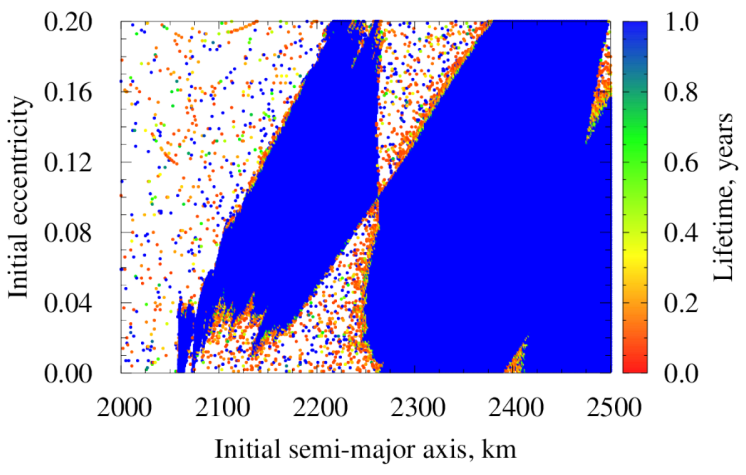

(b) $\theta_{\text {res }}=270^{\circ}$

Figure 2. Lifetime of the particles around Haumea as a function of the initial semimajor axis and eccentricity. Initial inclination $I=0.001^{\circ} . \varpi_{0}=$ $0^{\circ}$ (a) and $\varpi_{0}=90^{\circ}$ (b). Maximum time of integration equal to $1 \mathrm{yr}$. The white region stands for orbits that escaped or collided with Haumea, and orbits that have initial periapsis radius inside Haumea.

and all perturbations are included for all maps. Different maps only illustrate different aspects of the same dynamics. PMaps are a relatively new concept and, in particular, the analysis of the PMaps of type II and IV is novel. Thus, to explain the structures that appear in these maps, we formulate an hypothesis and we verify based on the characteristics of the real ring as given by Ortiz et al. (2017) and by the comparison of our findings with results in the literature, specifically with Sicardy et al. (2018) and Winter, Borderes-Motta \& Ribeiro (2019).

\subsubsection{PMap of type II - Combined effect of all perturbations}

In Fig. 3, which presents the first PMap of type II for $\theta_{\text {res }}=0^{\circ}$ (a) and $\theta_{\text {res }}=270^{\circ}$ (b), various regions are apparent. These are regions with alternating values of $\mathrm{PI}_{\mathrm{ii}}$, regions with a great concentration of positive values of $\mathrm{PI}_{\mathrm{ii}}$, and those with structures like diagonal lines. Regions with alternating values of $\mathrm{PI}_{\mathrm{ii}}$ i.e., near orbits with distinct behaviours are an indication of chaos. These regions can be seen for orbits with high eccentricity near $a_{0}=2500 \mathrm{~km}$ in Fig. 3(a) and for orbits at the extremes along a diagonal line starting $a_{0}=2400 \mathrm{~km}$ in Fig. 3(b). Regions with positive high values of $\mathrm{PI}_{\mathrm{ii}}$ are separated into two classes. The first class is comprised of orbits that do not survive for the entire time of integration and the second class is comprised of orbits that survived for the total time of integration (see Fig. 2 for details on survival regions). In both cases, the positive high value indicates an increase in some orbital element. In the case of the first 


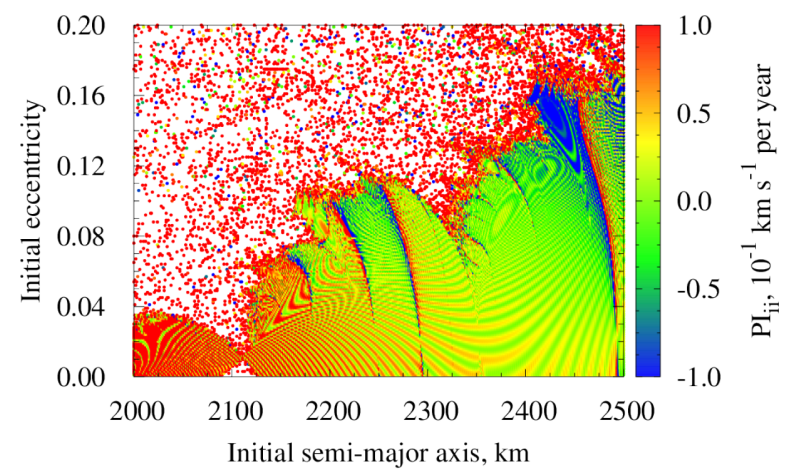

(a) $\theta_{\text {res }}=0^{\circ}$

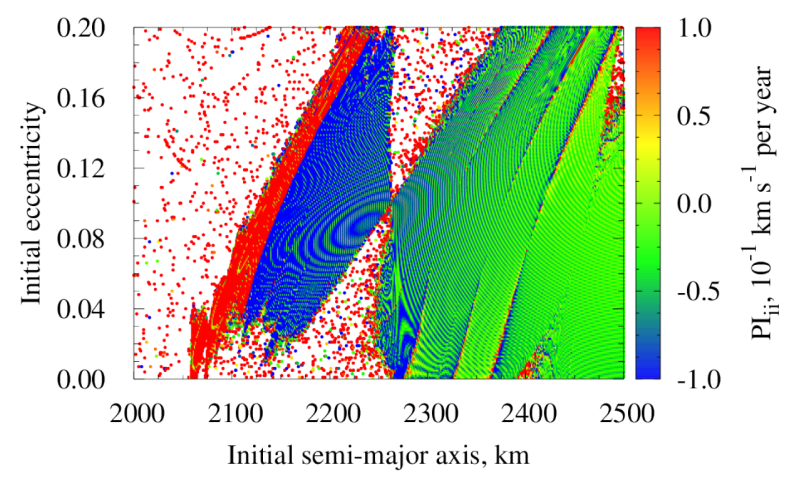

(b) $\theta_{\text {res }}=270^{\circ}$

Figure 3. Perturbation map of type II coming from Fig. 1. This map demonstrates the balance in the energy of particles around Haumea, as a function of the initial semimajor axis and eccentricity.

class, there were increases in eccentricity and semimajor axis, since those orbits escape from the system. In the case of the second class, the semimajor axis and the eccentricity do not increase. Meanwhile, in Section 3.4, we show that these high values of $\mathrm{PI}_{\mathrm{ii}}$ are related to sudden changes in the angular orbital elements.

The diagonal lines present two sides, one with positive values and the other one with negative values. This behaviour is similar to the separatrices for resonances in phase spaces, as apparent in the phase space of a pendulum, since separatrices indicate change in the regime of a motion. In the case of a pendulum, it indicates the change from circulation to libration, but in the case of the PMap of type II, a separatrix indicates a change from the tendency to increase the value of an orbital element (high positive values) to a likely decrease in the value (high negative values), or vice versa. The presence of separatrices in the PMap of type II only indicates the presence of resonances. Since the gravitational model used in the simulations incorporates the expansion in spherical harmonics up to order and degree four, some of the resonant terms may be present in more than one term of the potential. This leads to the superposition of resonances showed in Fig. 3, indicated by the presence of more than two separatrices in the same region. The observational data given by Ortiz et al. (2017) indicates that the particles of the ring are in the 3:1 resonance. The region between $a_{0} \approx 2050$ and $\mathrm{a}_{0} \approx$ $2250 \mathrm{~km}$ is actually a prominent separatrix, and the orbits on that region should not survive in long term integrations.

In the next section, the individual contribution of each harmonic is shown in PMaps of type II.

\subsubsection{PMap of type II - individual contribution of the harmonics}

The effect of the coefficients of each harmonic of the gravitational potential of Haumea, $C_{n m}$, where $n$ is the order and $m$ is the degree of the coefficient, can be seen by using separate PMaps for the harmonics with coefficients $C_{20}, C_{22}, C_{40}, C_{42}$, and $C_{44}$ (Section 2.1). To measure the importance of each harmonic, we need to analyse the number and size of structures that appear in the individual map, for a given interval of $\mathrm{PI}_{\mathrm{ii}}$. Comparing Fig. 4(a) with Fig. 4(b), for $\theta_{\text {res }}=0^{\circ}$, one can notice that the influence of the harmonic with coefficient $C_{20}$ is one order of magnitude smaller that the influence of the harmonic with coefficient $C_{22}$. In fact, the shape of Fig. 4(b) is very similar to the shape of Fig. 3(a), indicating that the $C_{22}$ is the dominant harmonic in the dynamics of the particles in the ring. It is also noticeable that the order of magnitude of the $C_{40}$ (Fig. 4c) and $C_{42}$ (Fig. 4d) are also smaller if compared with the harmonic $C_{22}$. However, the harmonic with coefficient $C_{44}$ (Fig. $4 \mathrm{e}$ ) is only one order of magnitude smaller than the $C_{22}$ and it has a prominent separatrix near $a_{0} \approx 2300 \mathrm{~km}$. This means that the mentioned separatrix, which appears as a thin line in Fig. 3(a), is due to a resonant term present in the harmonic with coefficient $C_{44}$. Then, the order of importance of the harmonics in the dynamics of the particles, from the more important to the less important is: $C_{22}$, $C_{44}, C_{20}, C_{42}$, and $C_{40}$.

When compared with the sectorial $\left(C_{n, m}\right.$, with $\left.n=m\right)$ and tesseral $\left(C_{n, m}\right.$, with $\left.n \neq m\right)$ harmonics of the gravitational potential of Haumea, it is expected that the zonal harmonics $\left(C_{n, 0}\right)$ have a smaller effect. This is due to the fact that all orbits are almost planar with respect to the equator of Haumea. Comparing Figs 4(a, $\mathrm{b}, \mathrm{c}, \mathrm{d}$, and e), one can notice that the orbits that escape from the system, identified by Fig. 1(a), have positive values in the PMap of sectorial and tesseral terms, and negative values in the PMaps of the zonal terms. Escapes from the system occur when the particles have a close approach with Haumea. Since Haumea is a prolate body and the orbits of the particles are almost planar, these escapes occur when a particle gets too close to the prolate part of Haumea.

All the discussion about the role of the coefficients in the dynamics of the particles in the ring made for the case with $\theta_{\text {res }}=0^{\circ}$ can also be applied for the cases with $\theta_{\text {res }}=270^{\circ}$, shown in Fig. 5. As for the case $\theta_{\text {res }}=270^{\circ}$, the separatrix of the resonance present in the term of the harmonic with coefficient $C_{44}$ is prominent, and appears near $a_{0} \approx 2350 \mathrm{~km}$. This confirms the importance of the $C_{44}$ term also for $\theta_{\text {res }}=270^{\circ}$. The order of importance of harmonics is also the same.

\subsubsection{PMap of type IV}

PMap of type IV shows how much the orbit of a particle deviates from a reference orbit. We use, for each initial condition, a Keplerian orbit as the reference orbit. Therefore, the PMap of type IV indicates if the particles have stable or unstable tendencies. If the orbits of the particles deviate too much from the reference orbit, i.e., the particles have high values of $\mathrm{PI}_{\mathrm{iv}}$, one can conclude that these orbits are unstable, having a low chance to stay around Haumea. On the other hand, orbits with low or almost zero values of $\mathrm{PI}_{\mathrm{iv}}$ could be stable. Additionally, as seen in Sanchez \& Prado (2019), periodic orbits would appear with near zero values in both PMaps of type II and IV. However, there is an exception to these rules: resonances. When in resonance, the orbit will never have near zero values of $\mathrm{PI}_{\mathrm{iv}}$. Instead, the map will present a 'wavy' shape, due to the resonant effect, with non-zero values of $\mathrm{PI}_{\mathrm{iv}}$. Due to this fact, no periodic orbit was identified in the system, as suggested by Winter et al. (2019). 


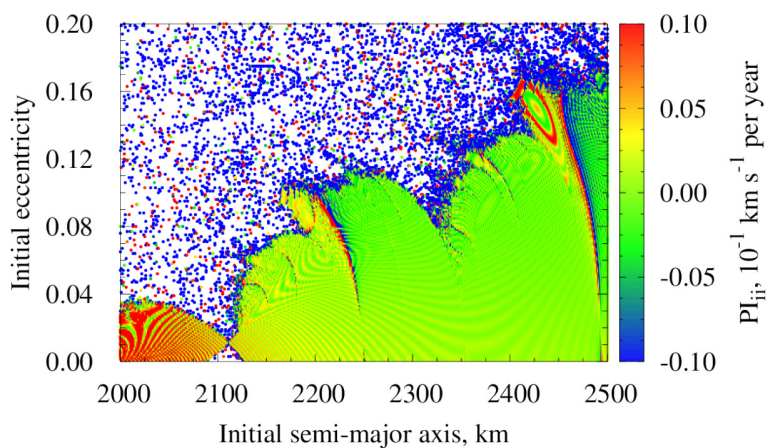

(a) $C_{20}$

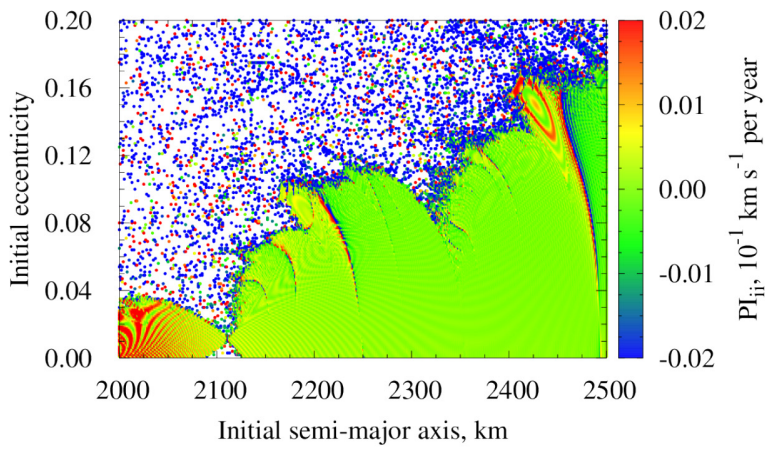

(c) $C_{40}$

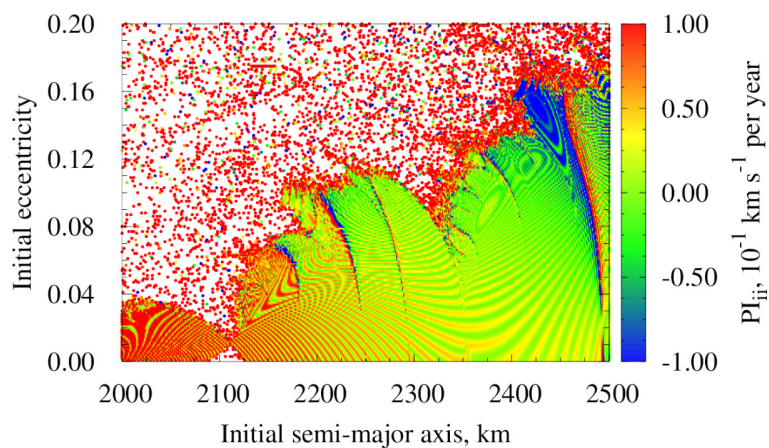

(b) $C_{22}$

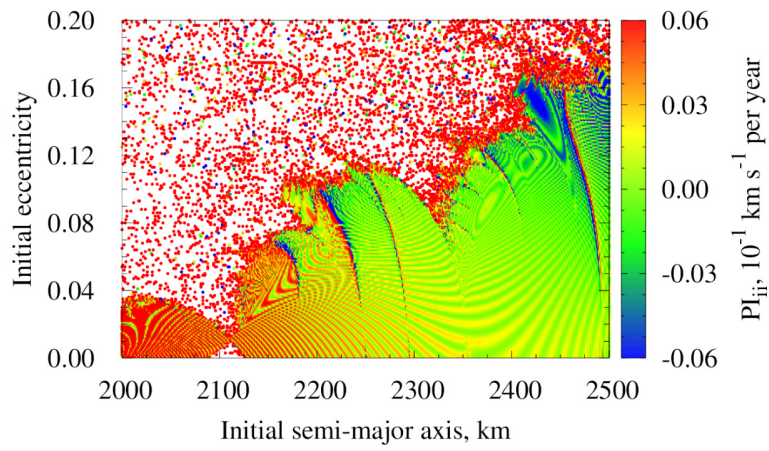

(d) $C_{42}$

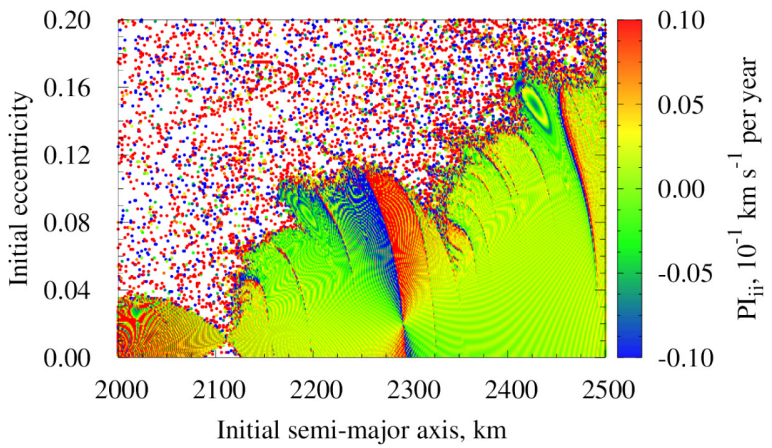

(e) $C_{44}$

Figure 4. Perturbation maps of type II coming from Fig. 1. Each map presents the individual contribution of one harmonic for $\theta_{\text {res }}=0^{\circ}$.

The regions with the maximum values of $\mathrm{PI}_{\mathrm{iv}}$ as shown by the map follow the lines of the separatrices presented in Fig. 3. This pattern is expected because the line that separates two distinct behaviours should deviate more from the reference orbit. The consequence of this deviation is a spike in the eccentricity of the ring on these lines, affecting the ring structure. This observation will be discussed in the next section. Furthermore, the red lines in a ' $v$ ' shape in Fig. 6(b), with vertex near $a_{0} \approx 2150 \mathrm{~km}$ and $e_{0} \approx 0.04$, indicates that this region is possibly not stable.

\subsection{Considerations about the ring structure}

The presence of regions inside the ring that lead to increases in the orbital semimajor axis, as well as increases in the eccentricity of these particles, suggest that the ring has substructures. As mentioned before, this paper does not consider the interaction between the particles. However, regardless of the interaction between particles, any increase in semimajor axis and/or eccentricity in regions of the ring could lead to gaps. Thus, based on our findings, we can think of two scenarios that are worth attention. One is that Haumea may have a system of rings, instead of a single one. Another possibility is that the separatrices could limit the ring, since any particle that crosses these lines has an increase of eccentricity. Therefore, the findings in this paper provide interesting scenarios for the formulation of theories about the formation of the ring, as well as its final state (we leave this for future investigation).

It is also worth mentioning a work in which particle interactions were considered (Sicardy et al. 2018). In such work, Sicardy et al. (2018) have shown the strong coupling between Haumea and a surrounding collisional disc, putting tight constraints on the final location of the ring. Sicardy et al. (2018) have shown that the 2:1 resonance between the mean motion of the particles and the rotation 


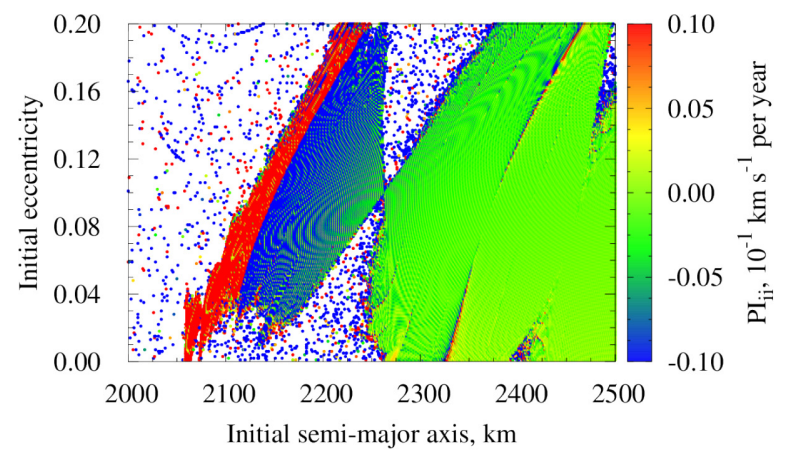

(a) $C_{20}$

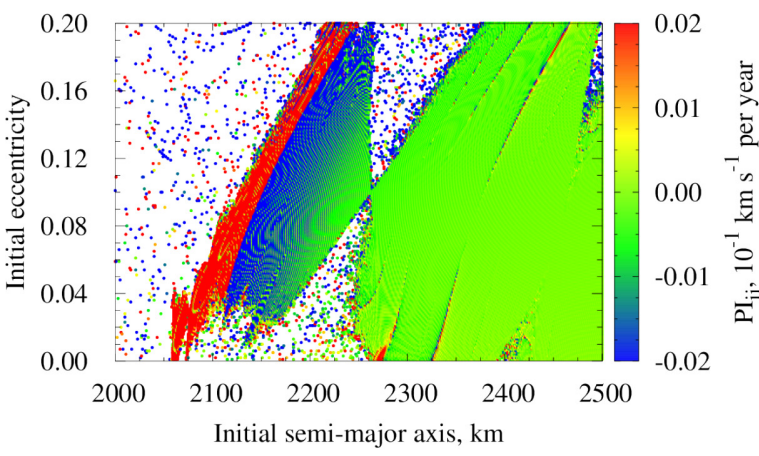

(c) $C_{40}$

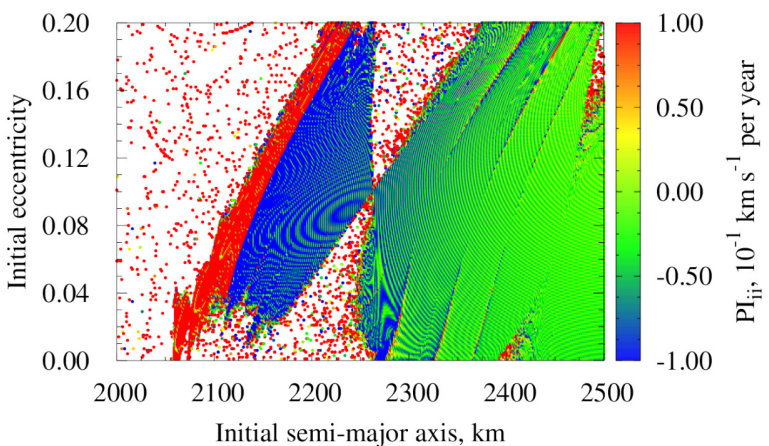

(b) $C_{22}$

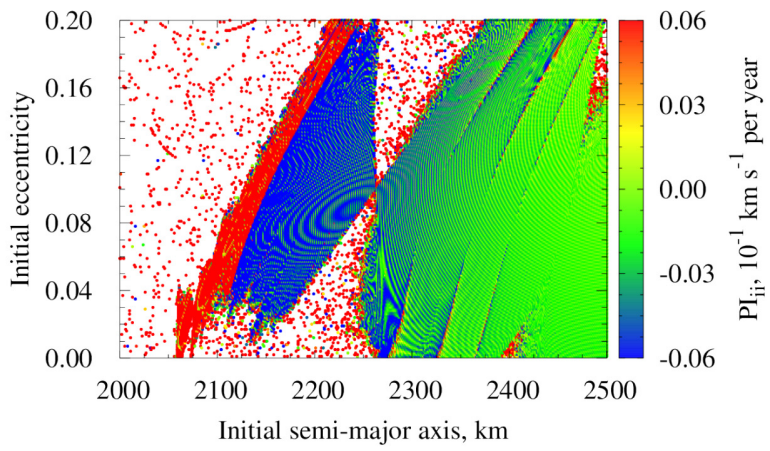

(d) $C_{42}$

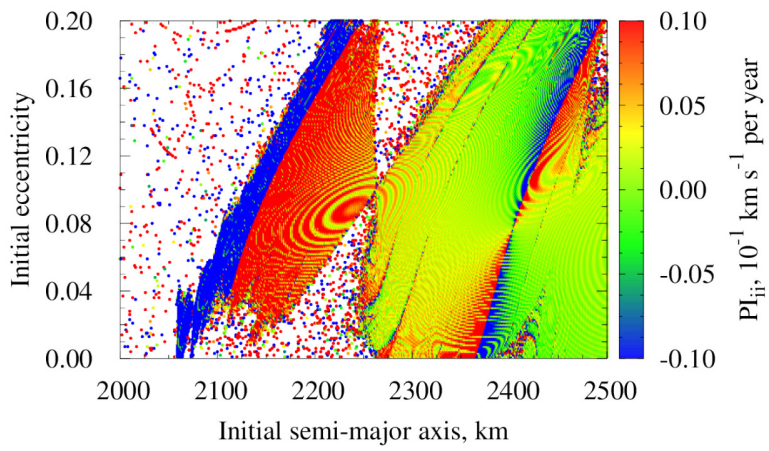

(e) $C_{44}$

Figure 5. Perturbation maps of type II coming from Fig. 1. Each map presents the individual contribution of one harmonics for $\theta_{\text {res }}=270^{\circ}$.

of Haumea leads the particles to fall to the surface of Haumea. Although using completely different methods, our results confirm the absence of particles within the region where the resonance 2:1 is supposed to be, at $a_{0}=1758.4808 \mathrm{~km}$.

The whole study of the PMap considers integrations over a maximum period of $1 \mathrm{yr}$. One of the advantages of the PMaps is that they can provide information in short periods of integration. However, to confirm the results apparent up to now, we perform additional simulations where we set the total integration time to 1000 yr. Hi' iaka and Namaka are also present in the simulations. The $1000 \mathrm{yr}$ integration time was chosen due to the fact that the longitude of the ascending node of Hi'iaka (the slowest evolving variable) has a period of about $800 \mathrm{yr}$. Therefore, we can assure that all possible geometrical configurations of the system will be covered by this integration time. We consider simulations with initial eccentricity $e=0.005$ and initial inclination $I=0.001^{\circ}$, varying the initial semimajor axis with the same range of the grids of initial conditions previously presented.

Fig. 7 shows the maximum orbital radius (top panel) achieved by the orbits of particles around Haumea from the integration performed, as a function of the semimajor axis, for $\theta_{\text {res }}=0^{\circ}$. Detailed view of the lower values of the maximum orbital radius (centre panel of Fig. 7) and the minimum orbital radius (bottom panel of Fig. 7) achieved are also plotted. In this figure, we can see that there are two clear unstable regions that match with the findings in Fig. 6(a). These two regions could limit the size of the ring, since the ring was observed between these two regions. As for the particles within the remaining regions, although stable, some of them present significant variations in their orbital radius as well. The regions with increases in the orbital radius are exactly the same points where Fig. 6(a) showed high values of $\mathrm{PI}_{\mathrm{iv}}$. This means that the PMap successfully predicted long-term behaviour of the 


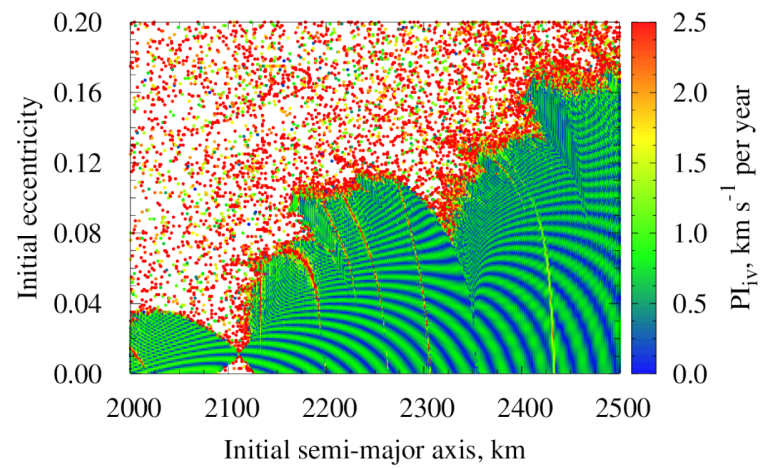

(a) $\theta_{\text {res }}=0^{\circ}$

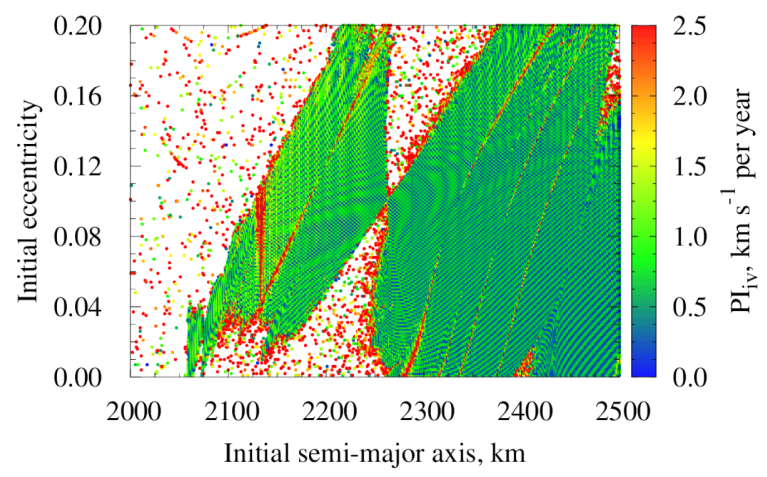

(b) $\theta_{\text {res }}=270^{\circ}$

Figure 6. Perturbation map of type IV coming from Fig. 1. This map shows how much the orbit of a particle deviates from a Keplerian reference orbit around Haumea, as a function of the initial semimajor axis and eccentricity.

particles. Also, if the particles could interact among themselves, and collisionaly evolve, those spikes in the orbital radius could represent the source for a gap opening inside the ring. If considering $\theta_{\text {res }}=$ $270^{\circ}$ (Fig. 8), the stable region is smaller than the stable region in Fig. 7, but compatible with Fig. 6(b). Therefore, the long-term integrations confirm the information from the PMaps.

By the analysis of the escape mechanism of each orbit in our simulations, we noticed that some of the particles leave the ring region, crossing the orbit of Namaka, passing by the chaotic region between Haumea and Namaka (Sanchez \& Prado 2017). In this escape mechanism, the orbital eccentricity of the particle grows due to its interaction with Haumea during close encounters. This can be observed by checking the values of the minimum orbital radius of the particles, in the case of escapes, in Figs 7 and 8. Therefore, when crossing the chaotic region between Haumea and Namaka, particles can reach any place in the system before escaping. Particles may also cross the orbit of Namaka. In this case, they may become temporary eccentric co-orbitals (Sanchez \& Prado 2017). Since these co-orbital orbits are not stable, the particles can return to the ring region and eventually colliding with Haumea (Fig. 9). Fig. 9 shows the trajectory (a) of a particle (red) that collides with Haumea and its projections on the planes $x y$ (b), $x z$ (c), and $y z$ (d), after crossing the orbit of Namaka (green). This trajectory has initial semimajor axis equal to $2296.3955 \mathrm{~km}$, initial eccentricity equal to 0.11 and initial $\theta_{\text {res }}=0^{\circ}$. This semimajor axis is the theoretical semimajor axis for the 3:1 resonance (and consequently for the 6:2 resonance), which means that a particle with this semimajor axis will have a period three times larger than the rotational period of
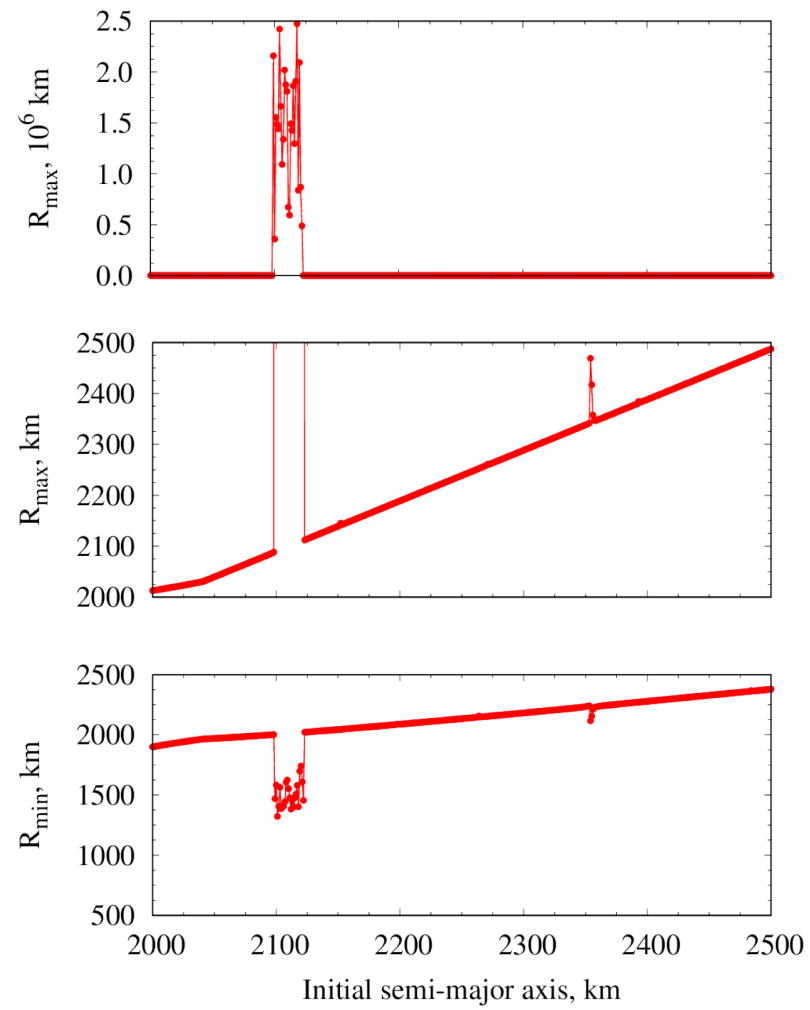

Figure 7. Maximum radius achieved by the orbits of particles around Haumea for a maximum time of integration of $1000 \mathrm{yr}$ (top) as a function of the semimajor axis. Detailed view of the lower values of the maximum radius (centre). Minimum radius achieved (bottom). These figures have initial eccentricity $e=0.005$, initial inclination $I=0.001^{\circ}$, for $\theta_{\text {res }}=0^{\circ}$.

Haumea. It is clear in Fig. 9 that, after a close passage by Haumea, the particles can reach large values of orbital eccentricity, with apoapsis radius close to the value of the semimajor axis of Namaka. This mechanism also creates the possibility of a collision of the particle with Namaka, although small, due to the size of that moon. The possibility that the particle stays, temporarily, as a co-orbital of Namaka is interesting, because this opens the possibility of the transit of particles between Haumea and the orbit of Namaka. This transit may be a continuous process, and indicates that the vicinity of Namaka may not be empty.

\subsection{Considerations about the resonances in the ring}

If we consider the information coming from Figs 7 and 8 alone, there is a false impression that the particles of the ring have a preferential non-3:1-resonant configuration, since the ring with initial $\theta_{\text {res }}=0^{\circ}$ is larger than the ring with initial $\theta_{\text {res }}=270^{\circ}$, which is the centre for the 3:1 resonance. However, as we can see in Fig. 3(a), a large part of the figure is dominated by positive values of $\mathrm{PI}_{\mathrm{ii}}$, which indicates changes in the angular orbital elements of the particles, as mentioned before. This phenomenon will be discussed in this section.

To make conclusive assumptions about the resonance of the ring, we decided to see what happens with the resonant angle in the first days of the integration. Fig. 10 shows the time evolution of the resonant angle for several initial values of eccentricity, considering the theoretical value for the 3:1 spin-orbit resonance semimajor axis, $a_{\text {res }}=2296.3955 \mathrm{~km}$, as the initial semimajor axis. In Fig. 10(a) it 

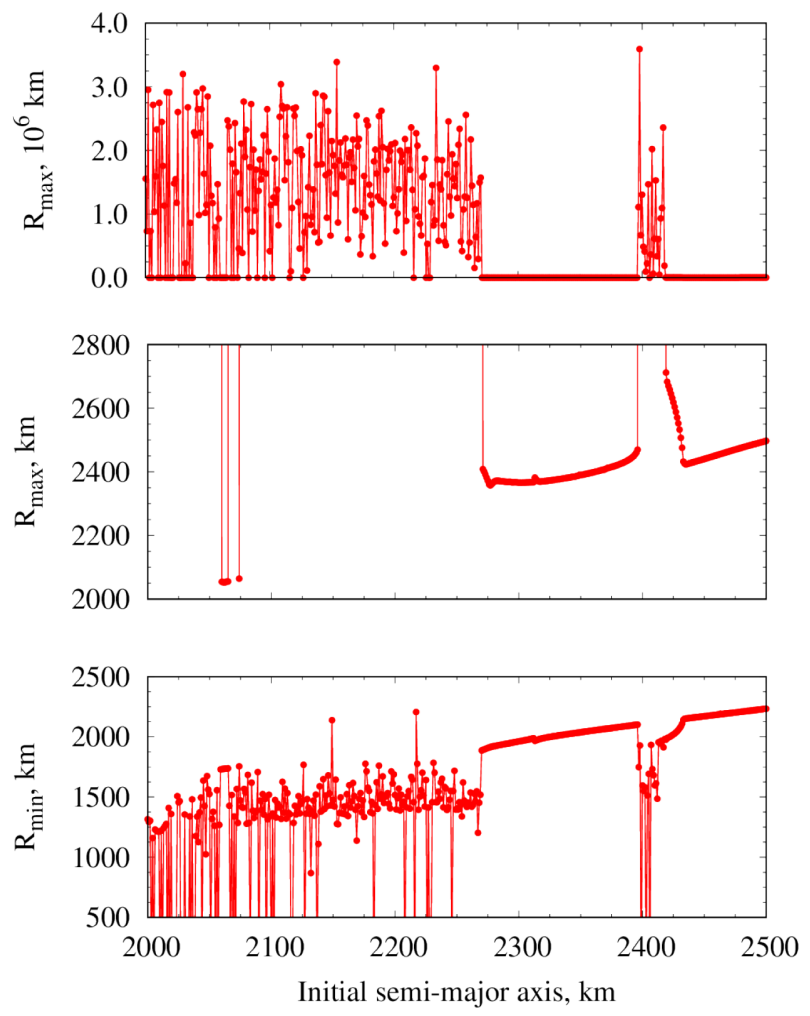

Figure 8. Maximum radius achieved by the orbits of particles around Haumea for a maximum time of integration of $1000 \mathrm{yr}$ (top) as a function of the semimajor axis. Detailed view of the lower values of the maximum radius (centre). Minimum radius achieved (bottom). These figures have initial eccentricity $e=0.005$, initial inclination $I=0.001^{\circ}$, for $\theta_{\text {res }}=270^{\circ}$.

is possible to assume that the resonant angle is in libration around $0^{\circ}$, with a large amplitude, for high values of eccentricity. The amplitude increases with the decrease in eccentricity, until the angle starts to circulate for $e_{0}<0.08$. However, if we consider that Haumea is a fast rotating ellipsoid, with a rotation period of $3.1955 \mathrm{~h}$, one can consider that part of the resonant mechanisms may be present for periods of time within the same order of magnitude, or even smaller than the rotational period of Haumea. In this way, it is possible to observe, in Fig. 10(a), that there are libration-like structures around $\pm 90^{\circ}$ that occur for very short times, during the circulations. For example, in the first circulation for $e_{0}=0.04$, the resonant angle 'stays' for $0.08 \mathrm{~d}$ (half of Haumea's period) around $90^{\circ}$ and then returns to the circulation; stops again around $270^{\circ}$, also for $0.08 \mathrm{~d}$, then resume circulation. These short time librations that we called pseudo-librations, are indicative of the presence of the 3:1 resonance, although there is no capture in neither of the resonant angles. Furthermore, the libration period around $0^{\circ}$ for the higher values of eccentricity is commensurable with the period of Haumea, in the ratio of 3:1 between the rotation of Haumea and the libration period. This is another indication of the presence of the 3:1 resonance.

If the time of integration for Fig. 10(a) is extended for $1000 \mathrm{yr}$, all the orbits that survive for the new time of integration are the ones with pseudo-librations, that occur for $e_{0}<0.08$, as it can be seen in Figs $11(\mathrm{a}-\mathrm{c})$. For higher values of eccentricity, whose resonant angle is present for libration around $0^{\circ}$, the orbits do not survive for $1000 \mathrm{yr}$ and are classified as unstable. This can be seen in Fig. 11(d). In this figure, after a few days with libration around $0^{\circ}$, the resonant angle starts to circulate (without the pseudo-librations) and then the particle escapes from the system. In fact, this is the resonant angle for the orbit presented in Fig. 9. The transition between the libration to the circulation regime happens at approximately 0.005 yr $(\sim 1.8 \mathrm{~d})$, when a close passage by Haumea occurs. After this passage, there is an increase in semimajor axis value and the particle leaves the 3:1 resonance and, consequently, the resonant angle starts to circulate. The libration around $0^{\circ}$ enables the particle to pass close to Haumea in the direction of the 'tips' of Haumea $(\omega=$ $\left.0^{\circ}, \omega=180^{\circ}\right)$. In this way, close encounters in this configuration generate large increases in the semimajor axis. This observation could indicate that this configuration is unstable. Furthermore, these increases in the semimajor axis allow the particle to reach Namaka's orbit, making possible the transit of particles between Haumea and Namaka.

As mentioned before, in Fig. 3(a) there is a great number of high positive values of $\mathrm{PI}_{\mathrm{ii}}$, but without escapes. These orbits present positive values of $\mathrm{PI}_{\mathrm{ii}}$ due to the presence of the pseudo-librations inside the circulation regime. The variation of the resonant angle is not constant: the pseudo-librations are, actually, sudden decreases in the variation ratio of the resonant angle. Recall that Haumea as an ellipsoid and that its rotation period is three times the period of the particles in the ring, these decreases in the variation ratio of the resonant angle occur when particles are passing by the sides of Haumea, at $\omega=90^{\circ}$ and $\omega=270^{\circ}$. This is the direct effect of the $C_{22}$ term of the gravitational potential of Haumea, which gives to Haumea the prolate shape. Although this is the dominant term, as it demonstrated in the harmonics analysis, the harmonic with coefficient $C_{44}$ is also important and could prevent the resonance to lock in $\theta_{\text {res }}= \pm 90^{\circ}$.

In the case of initial resonant angle $\theta_{\text {res }}=270^{\circ}$, in Fig. 10(b) and in the set of plots in Fig. 12, there is no commensurability between the period of the resonant angle and the rotation of Haumea. Consequently, for the semimajor axis analysed, the resonant angle is circulating for all values of initial eccentricity. Furthermore, the variation ratio of the resonant angle is not constant and the pseudolibrations are also present. For Fig. 10(b) there was a preferential value of eccentricity where the pseudo-librations lasted for longer $(e=0.04)$. In the case of $\theta_{\text {res }}=270^{\circ}$, the preferential value of eccentricity is 0.08 , double of the correspondent value for initial $\theta_{\text {res }}=0^{\circ}$. This observation may explain why there are more stable orbits for high eccentricities for $\theta_{\text {res }}=270^{\circ}$, if comparing Figs 3(a) and (b).

For the initial resonant angle $\theta_{\text {res }}=270^{\circ}$, escapes occur for values of eccentricity for which the resonant angle is in the circulation regime (Fig. 12d), but without libration in $0^{\circ}$.

Regarding the eccentricity of the ring, even beginning with near zero eccentricity, all particles achieve considerable values of eccentricity after some time of integration. This can be observed in both Figs 7 and 8, by comparing the values of the minimum and maximum orbital radius. However, this result does not mean that the real ring has high values of eccentricity. Part of the eccentricity presented by the particles can be induced by the use of osculating elements instead of geometrical elements (Renner \& Sicardy 2006) for the conversion from Cartesian coordinates to orbital elements, and vice versa. Since we are using the expansion in the spherical harmonics of the gravitational potential of Haumea up to order and degree four, it is not possible to use geometrical elements, because these elements only correct the coefficient $J_{2}$. Currently, there are no geometrical elements for expansions of orders greater than two and this is an open problem. However, if the spikes in eccentricity occur above the systematic error in eccentricity caused by the orbital 


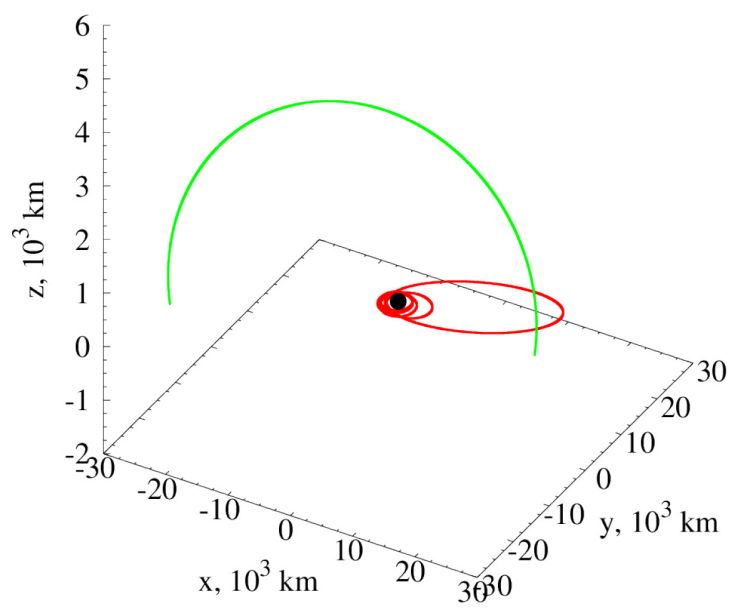

(a)

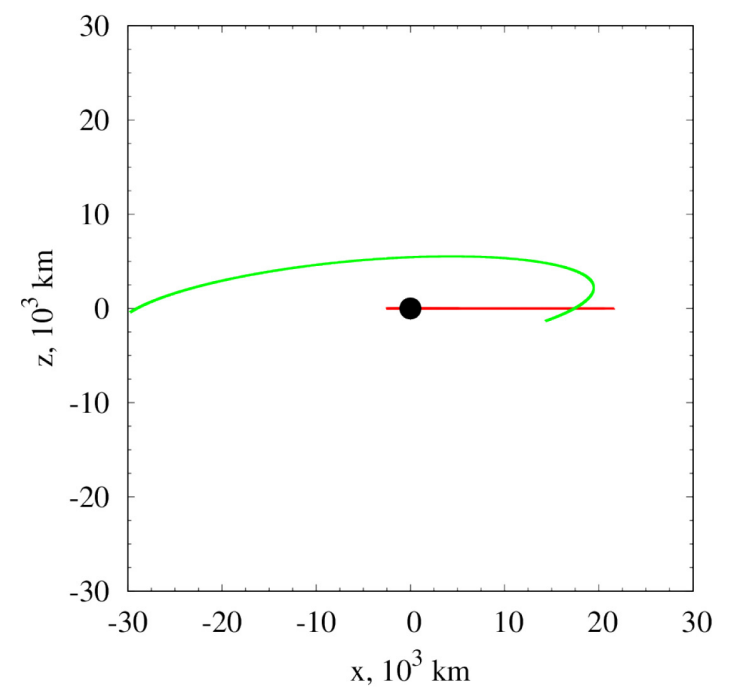

(c)

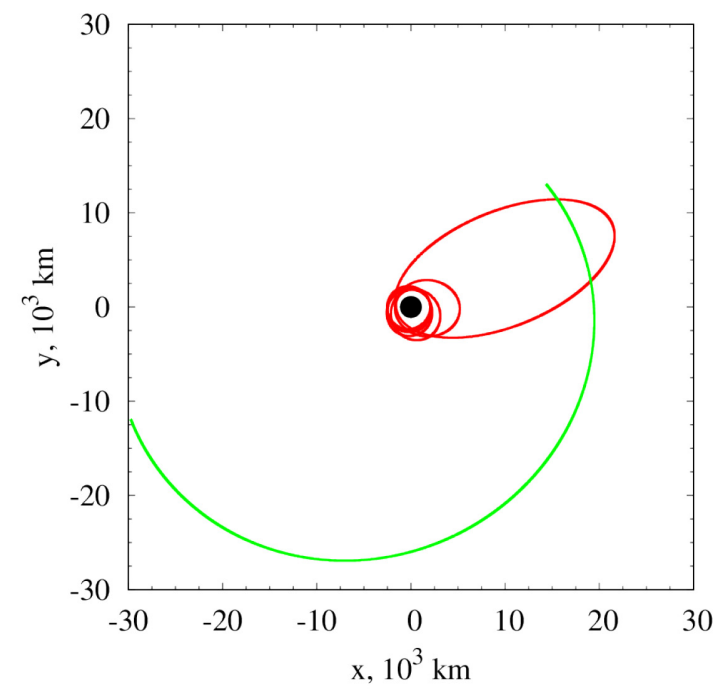

(b)

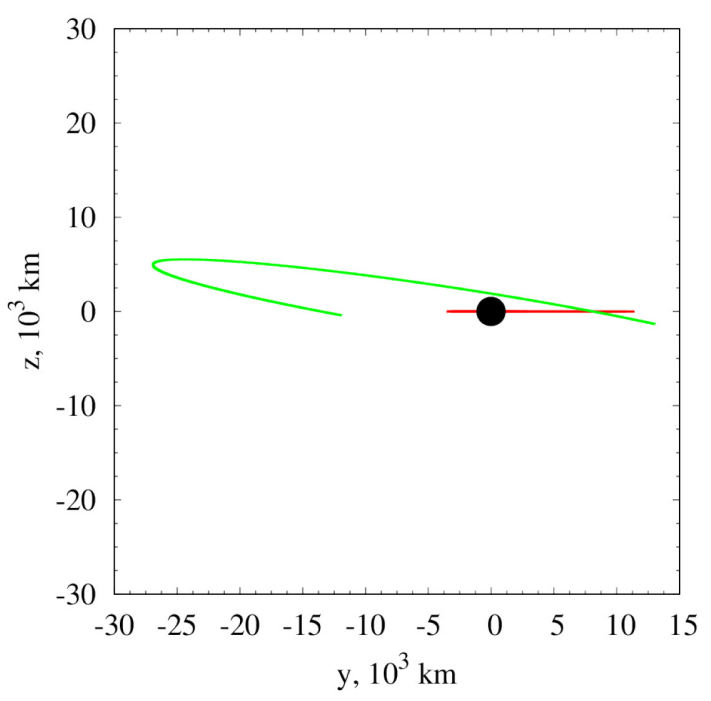

(d)

Figure 9. Trajectory of a particle, in red, (a) and its projections on the planes $x y$ (b), $x z$ (c), and $y z$ (d). Initial semimajor axis equal to $2296.3955 \mathrm{~km}$, initial eccentricity equal to 0.11 for initial $\theta_{\text {res }}=0^{\circ}$. The orbit of Namaka if shown in green.

elements conversion, then they are indeed effects caused by the resonances apparent in the PMaps, and the consequences of the existence of these spikes are real.

The semimajor axis used in this section was calculated using the two-body approximation, for the same reasons explained in the previous paragraph. However, we made a search in the vicinity of this semimajor axis to find the exact semimajor axis to which the resonance is locked in $\theta_{\text {res }}= \pm 90^{\circ}$. However, we did not determine such value of semimajor axis. The reason is the presence of the harmonics above $C_{22}$, mainly the harmonic $C_{44}$, as mentioned before.

\section{CONCLUSIONS}

The ring of Haumea was numerically investigated using PMaps of type II and IV. These maps provide information, using a short period of integration, that is not available from the usual grid of initial conditions, such as maps of the lifetime of the particles and the maximum eccentricity achieved. PMaps of type II showed that there are superposition of resonances coming from different terms of the gravitational potential of Haumea, expanded in spherical harmonics up to order and degree four. In this case, the dominant term is the one that contains the coefficient $C_{22}$, followed by the term with coefficient $C_{44}$. The analysis was completed for two values of the initial resonant angle for the 3:1 resonance, $\theta_{\text {res }}=0^{\circ}$ and $\theta_{\text {res }}=$ $270^{\circ}$.

The PMap of type II showed some structures in the region where the real ring was observed that resemble structures from dynamical systems, such as separatrices. Combining the results from the PMap of type II and the PMap of type IV, one can conclude that these separatrices induce gaps inside the region where the ring is constrained. Integrations for a period of $1000 \mathrm{yr}$ confirmed the findings given by the PMaps, showing that the places with high values of $\mathrm{PI}_{\mathrm{iv}}$ in the PMaps indicate regions with spikes in 


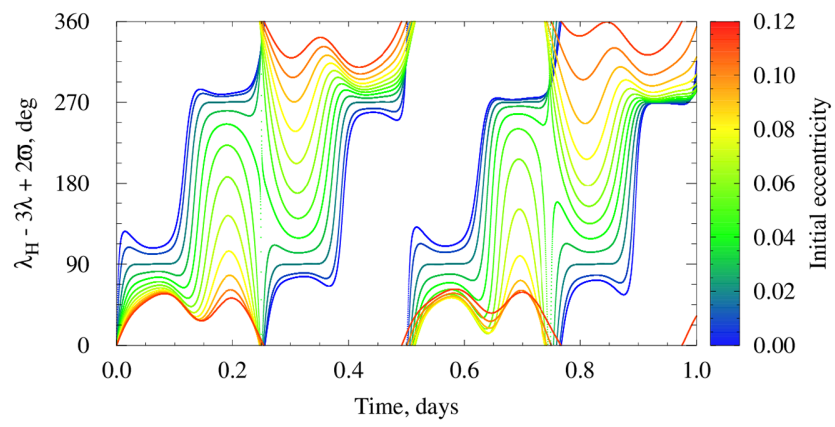

(a) $\theta_{\text {res }}=0^{\circ}$

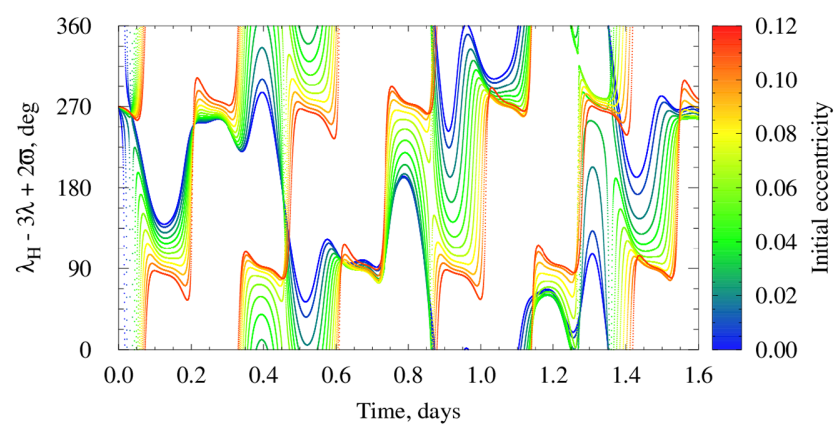

(b) $\theta_{\text {res }}=270^{\circ}$

Figure 10. Time evolution of the resonant angle for the first day of integration, for several values of initial eccentricity, for $\theta_{\text {res }}=0^{\circ}$ (a) and $\theta_{\text {res }}=270^{\circ}$ (b). Initial semimajor axis equal to $2296.3955 \mathrm{~km}$.

eccentricity. This analysis leads to the conclusion that the PMaps can be used to assess the stability of particles in a ring, using short times of integrations. Our results agree with observational data and previous work. It gives a new physical explanation for the rings that can be also applied to other systems.

Additionally, an analysis of the resonances present within the ring was also completed. In this case, it was shown that the 3:1 resonance is present in the dynamics of the particle. For the stable configurations of the ring, the resonant angle is circulating, but with no constant variation ratio. For $\theta_{\text {res }}=270^{\circ}$, there is sudden decrease in the variation ratio, resembling a very short period libration regime, which we call pseudo-librations. This is a strong indication that the $3: 1$ resonance is present, but not locked due to the presence of additional harmonics in the gravitational potential, mainly the harmonic with coefficient $C_{44}$. For $\theta_{\text {res }}=0^{\circ}$, the escapes of the particles from the ring aways occur after libration of large amplitude in $\theta_{\text {res }}=0^{\circ}$, with a period of three times the rotational period of Haumea. This libration allows the particle to make very close passages by Haumea, at $\omega=0^{\circ}$ and $\omega=180^{\circ}$, without a collision. These close passages induce increases in semimajor axis and eccentricity and, as a consequence, the particles to cross the unstable region between Haumea and Namaka. After this passage by the unstable region, the particle can escape from the system, colliding with one of the moons, or becoming temporarily captured as a co-orbital of Namaka. This scenario opens the possibility of transit of particles between Haumea and the vicinity of the orbit of Namaka. If a continuous process, this transit of particles would point towards a populated vicinity around Namaka. For $\theta_{\text {res }}=270^{\circ}$, the escapes occur for high values of eccentricity, typically twice the eccentricity value of the $\theta_{\text {res }}=0^{\circ}$ configuration.

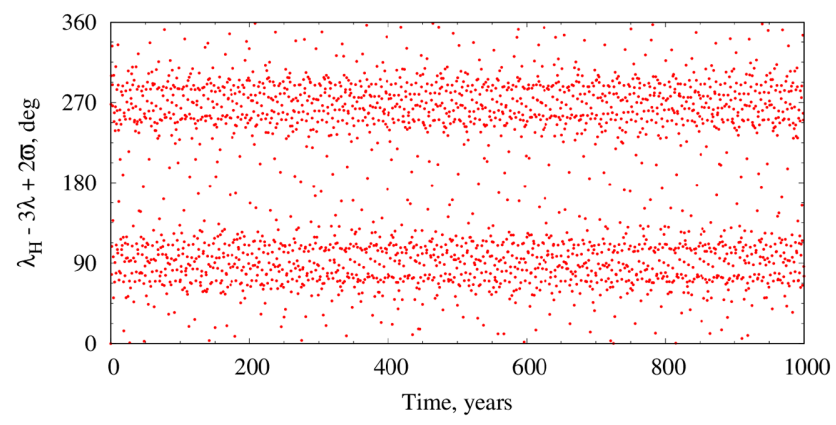

(a) $e=0.00$

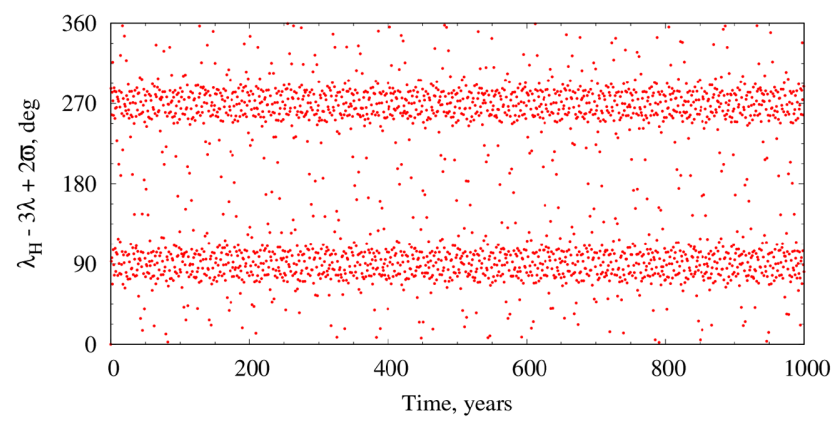

(b) $e=0.03$

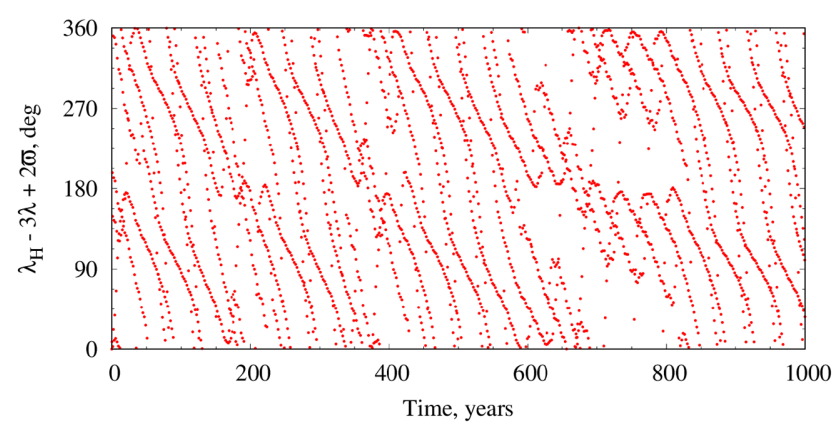

(c) $e=0.06$

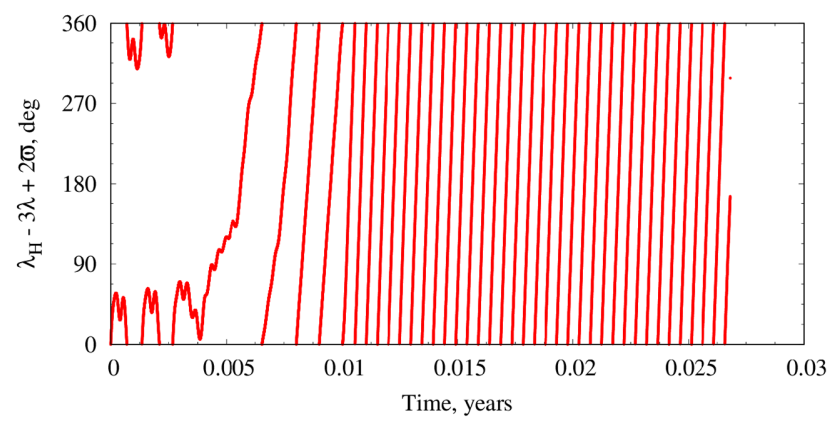

(d) $e=0.11$

Figure 11. Time variation of the resonant angle for four values of eccentricity. Initial resonant angle equal to $0^{\circ}$. Initial semimajor axis equal to $2296.3955 \mathrm{~km}$. 


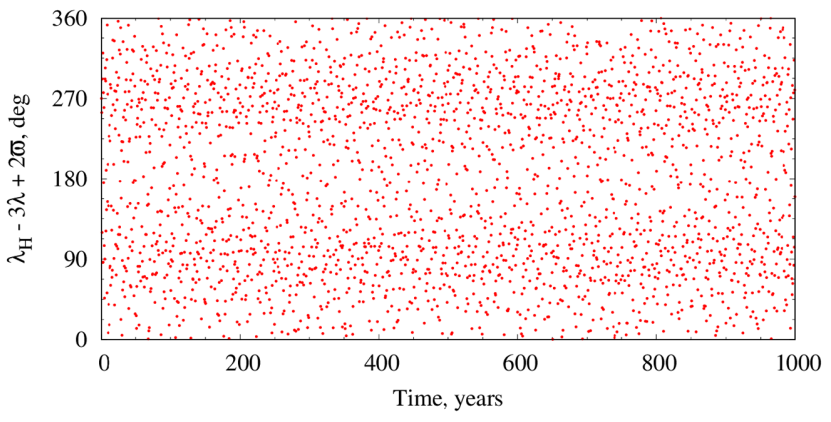

(a) $e=0.00$

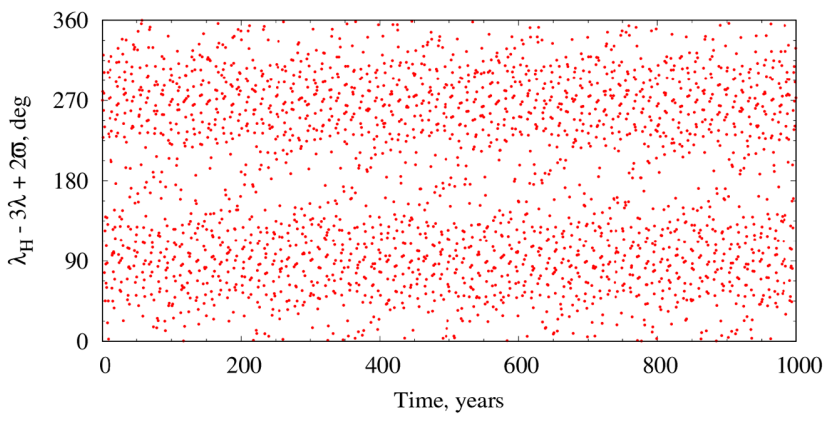

(b) $e=0.05$

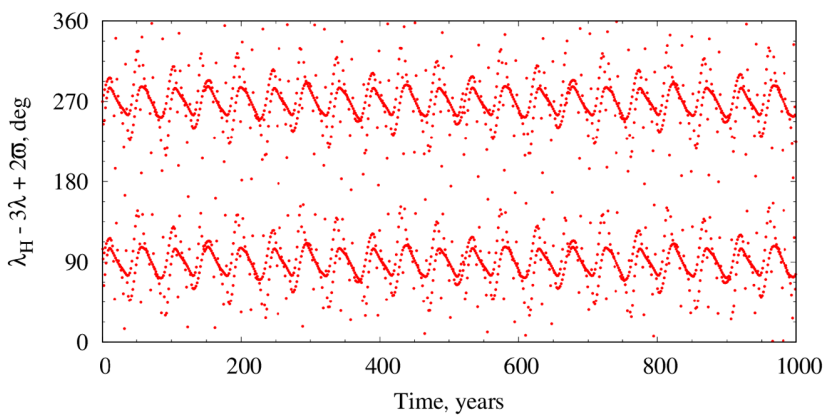

(c) $e=0.07$

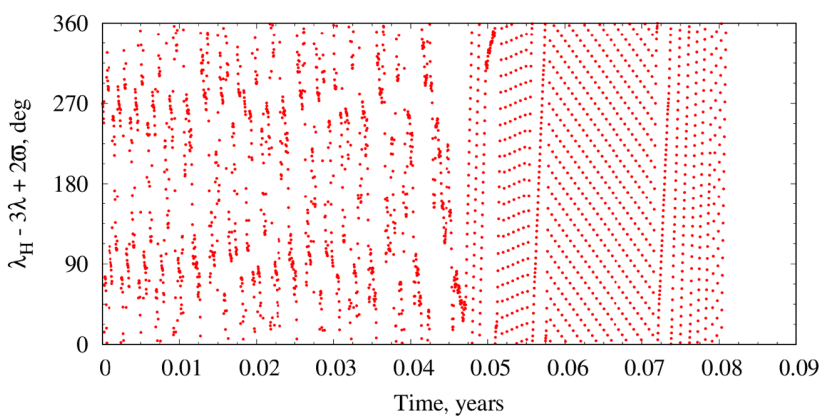

(d) $e=0.13$

Figure 12. Time variation of the resonant angle for four values of eccentricity. Initial resonant angle equal to $270^{\circ}$. Initial semimajor axis equal to $2296.3955 \mathrm{~km}$.

\section{ACKNOWLEDGEMENTS}

The authors wish to express their appreciation for the support provided by grants \#301338/2016-7 and 406841/2016-0 from the National Council for Scientific and Technological Development (CNPq) and grants \# 2014/22295-5 and 2016/24561-0 from São Paulo Research Foundation (FAPESP) and the financial support from the National Council for the Improvement of Higher Education (CAPES). The anonymous referees are gratefully thanked for their very useful comments.

\section{REFERENCES}

Balmino G., 1994, Celest. Mech. Dyn. Astron., 60, 331

Burden R. L., Faires J. D., 2010, Numerical Analysis. 9 edn., Cengage Learning, USA

Carvalho J. P. S., de Moraes R. V., Prado A. F. B. A., 2014, Math. Probl. Eng., 2014, 1

Ćuk M., Ragozzine D., Nesvorný D., 2013, AJ, 146, 89

Desch S. J., Neveu M., 2015, Proceedings.... Texas: The Woodlands. Universities Space Research Association, Lunar and Planetary Institute, Houston, TX, USA

de Almeida A. K., de Almeida Prado A. F. B., de Moraes R. V., Lara M., 2018, Comput. Appl. Math., 37, 7

dos Santos J. C., Carvalho J. P. S., Prado A. F. B. A., de Moraes R. V., 2015, J. Phys.: Conf. Ser., 641, 012011

Everhart E., 1985, in Carusi A., Valsecchi G. B., eds, Dynamics of Comets: Their Origin and Evolution. Springer Netherlands, Dordrecht, p. 185

Lara M., 2016, J. Guid. Control Dyn., 39, 2156

Levison H. F. et al., 2008, AJ, 136, 1079

Lockwood A. C., Brown M. E., Stansberry J., 2014, Earth Moon Planets, 111,127

Oliveira T. C., Prado A. F., 2014, Acta Astronaut., 104, 350

Ortiz J. L. et al., 2017, Nature, 550, 219

Pinilla-Alonso N., Brunetto R., Licandro J., Gil-Hutton R., Roush T. L., Strazzulla G., 2009, A\&A, 496, 547

Prado A. F. B. A., 2013, Math. Probl. Eng., 2013, 1

Prado A. F. B. A., 2014, Adv. Space Res., 53, 877

Ragozzine D., Brown M. E., 2009, AJ, 137, 4766

Renner S., Sicardy B., 2006, Celest. Mech. Dyn. Astron., 94, 237

Sanchez D. M., Prado A. F. B. A., 2017, Adv. Astronaut. Sci., 162, 1507

Sanchez D. M., Prado A. F. B. A., 2019, J. Spacecr. Rockets, 56, 1775

Sanchez D., Prado A. F. B. A., Yokoyama T., 2014, Adv. Space Res., 54, 1008

Sanchez D. M., Prado A. F. B. A., Yokoyama T., 2016a, Adv. Astronaut. Sci., 156, 1135

Sanchez D. M., Howell K. C., Prado A. F. B. A., 2016b, Adv. Astronaut. Sci., 158, 3681

Sanchez D. M., Howell K. C., Prado A. F. B. A., 2017, Adv. Astronaut. Sci., 160,215

Sicardy B., Leiva R., Renner S., Roques F., El Moutamid M., Santos-Sanz P., Desmars J., 2018, Nat. Astron.

Venditti F. C. F., Prado A. F. B. A., 2015, Mathematical Problems in Engineering, p. 11

Winter O. C., Borderes-Motta G., Ribeiro T., 2019, MNRAS, 484, 3765

This paper has been typeset from a $\mathrm{T}_{\mathrm{E}} \mathrm{X} / \mathrm{L} \mathrm{T} \mathrm{E} \mathrm{X}$ file prepared by the author. 